\title{
EXPERIMENTAL STUDIES - BUILDING PHYSICS INVESTIGATIONS IN CONDENSATION WATER ON PLASTER SURFACES
}

\author{
Torsten STEFFGEN ${ }^{*}$ \\ University of Applied Sciences, Faculty of Engineering, Wismar, Germany and \\ Marcel Breuer Doctorate School, Faculty of Engineering and Information Technology \\ University of Pecs, Boszorkany u. 2, 7624 Pecs, Hungary \\ e-mail: tsteffgen@gmx.de
}

Received 31 December 2017; accepted 8 October 2018

\begin{abstract}
In recent years, surfaces of plaster facades increasingly have been covered with algae. This effect is reinforced by improved thermal insulation of the building.

Envelope e.g. due to the use of plastics, as well as the climate change and lower air pollution. The accumulation of condensation water is therefore of decisive importance.

So far, the amount of condensation water was determined with static weight methods. New developments in material research attempt to illustrate the susceptibility to condensation with different measurement methods. The differences can be seen in various systems and materials. Developing a model representation for the future planning of facades is thus possible for new and the existing building. This model can be used to derive new characteristics for new facade design.
\end{abstract}

Keywords: Algae growth on facade, Condensation water on plaster

\section{Introduction}

In the past only the possibility of blow rain safety of facades was discussed, while at the same time, the condensation load was simply ignored. Different authors had advised on the problem with condense water on the façade, for example H. Venzmer and J. von Werder. They performed research using after a demanding measurement method. They realized that the gravimetric determination methods of using a suction fleece with a balance scale can deliver good results. However, the question is when exactly the right time determining the amount of condensate with a fleece and a spring balance is. How

\footnotetext{
${ }^{*}$ Corresponding Author
} 
can establish a new measurement method in a new developed experimental set-up? The following methods can lead to these first observations and findings:

- Contact angle measurement;

- Water absorption coefficient measurement (W-value);

- Condensation water measurement with;

- Balance scale with suction fleece;

- Laser Scanning Microscope (LSM).

\section{Protective function of the facade}

In the last few years in new constructions as well as in renovation of the building stock, more and more plastics have been used in projects on the facade for example thermal insulation to reduce the energy loss [1, pp. 67]. Thermal insulation of the building is more and more important for the architect and energy designer [2, pp. 61], $[3$, pp. 1-38] to reducing the greenhouse effect.

The United Nation Environment Program detected that buildings consume about $40 \%$ of global energy [4]. Many scientists who research on new building energy design are favor of passive houses to reduce carbon dioxide [5, pp. 2-4], [6, pp. 1-16].

The trend is towards more synthetic material for the insulation as well as for plasters and paint coatings. Von Werder as well as others has analyzed the complex range of problems of algae growth relating to the condensate load on facade [7, pp. 225]. Further research has found out that the monthly condensation time is up to 240 hours; the impact duration of rain per month, however, is only about 22 hours [8, pp. 233]. KalaniI and Krus from Fraunhofer Institution in Holzkirchen (Germany) have indicated that the condensation time is more than 10 times higher than the rainwater time [9, pp. 29-51].

This investigation allows the conclusion that moisture films on the surface of the components are one of the main factors for the growth of algae. Today scientists use static measurement methods to determine the actual amount of condensation directly on the surface by means of a fleece and a spring balance. In own experiments also detected differences in various climate and location-dependent factors. A standardized recording might help to make measurements more comparable.

Some manufacturers try to prevent the effects of algae growth on plaster and paint with targeted biocides (encapsulated or non-encapsulated). Biocides are chemical or biological substances used in Germany and Switzerland specifically to protect coatings and plasters against microbial degradation or algae growth. These agents do not remain in the facade, but can be washed out by rain and enter the groundwater and drinking water. The leaching of biocide active substances from plasters and paints have already been investigated and scientifically proven [10, pp. 7-10].

Biocides are only effective in the facade matrix, in the aqueous phase, when they reach to the target organisms. These biocides are washed out of the plaster each time it rains or condensation occurs [10, pp. 3-7]. Afterwards they enter the sewer system or groundwater [10, pp. 18-19]. 


\section{Measurement}

The classic procedure for testing of water condensation by dabbing is with suction fleece and weighing by means of a balance scale in a field trial while making measurements and results. However, this is strongly dependent on the location and time of year. The 'right' time (maximum of condensation water) must be chosen out to perform the weighing operation. The following measuring methods appear useful from the perspective of the author to gain further insight. Initial results are presented in this report, though these have yet to be verified. The plaster samples were manufactured with bagged cargo dry plaster. The samples have a diameter of $100 \mathrm{~mm}$ and a size of $10 \mathrm{~mm}$. The surface was constructed with a spatula in a slice structure.

Contact angle measurement: A drop of water on a surface is lowered during the contact angle measurement and an image shot is made through a microscope. Subsequently an angular measurement can be taken of the surface by means of sample editing software. A clear distinction between mineral and organic-bound plasters can be determined. Mineral-bound plasters (M) have an angle of contact $<90^{\circ}$, organic plasters (O) have a contact angle $>90^{\circ}$, see Fig. 1 and Fig. 2 .

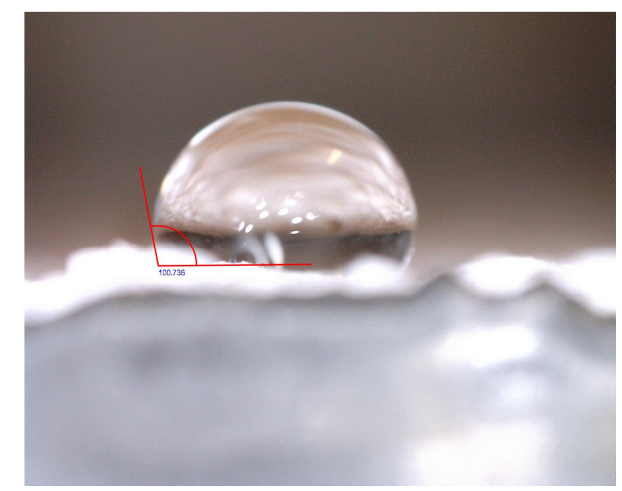

Fig. 1. Example of a contact angle measurement sample $\mathrm{O} 2$

A clear distinction can be determined between mineral samples M1 to M6 and organic-bound plasters $\mathrm{O} 1$ to $\mathrm{O} 6$. The mineral-bound plasters have an angle of contact $<90^{\circ}$, whereas the organic ones have a contact angle $>90^{\circ}$. Surface tension also varies considerably. If the contact angle is lower, the moisture penetration is deeper.

Water absorption coefficient ( $W$-value): The water absorption coefficient describes the technical behavior of building and construction material against heat and humidity heat and humidity according to EN ISO 151482002 [11], see Fig. 3. The water absorption due to capillary forces in partial immersion in a water bath can be evaluated with this method. This relates to temperature and moisture gradients and the properties of the respective building materials in a complex exchange relationship. The water absorption is determined by repeated weighing of the specimen at regular intervals over a period of 24 hours. The weight - increase - time diagrams in Fig. 4, Fig. 5 and Fig. 6 
indicate the trend. There is an example for organic plaster in Fig. 5 with low rise and an example for mineral plaster in Fig. 6 with high rise water absorption.

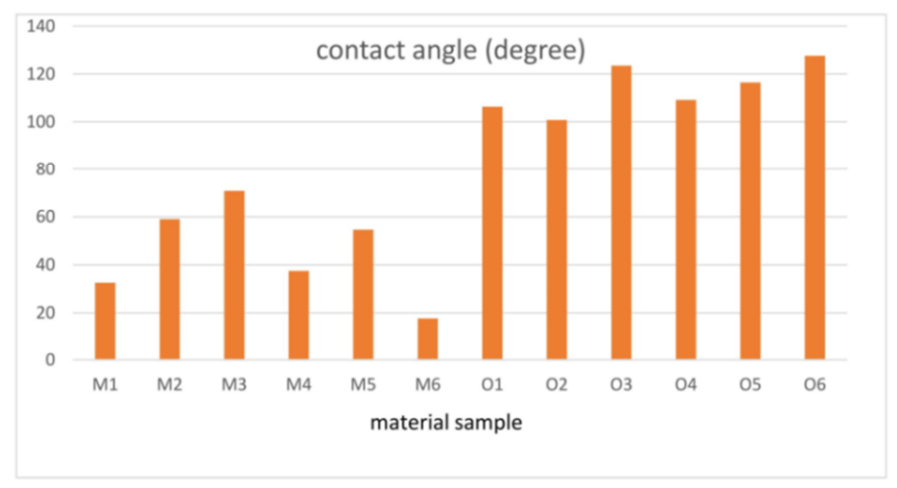

Fig. 2. Contact angle measurement of mineral plasters M 1 to $\mathrm{M} 6$, as well as organic plasters $\mathrm{O} 1$ to $\mathrm{O} 6$

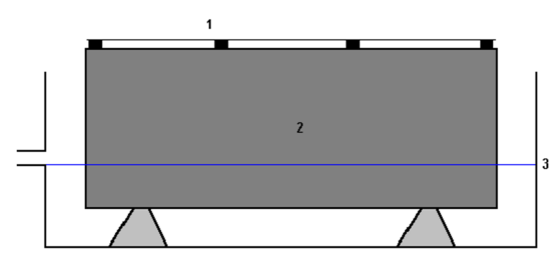

Fig. 3. Test arrangement of samples in accordance with EN ISO 15148, 2002 [12, pp. 8],

1. Surcharge (if necessary); 2. Sample; 3. Water surface

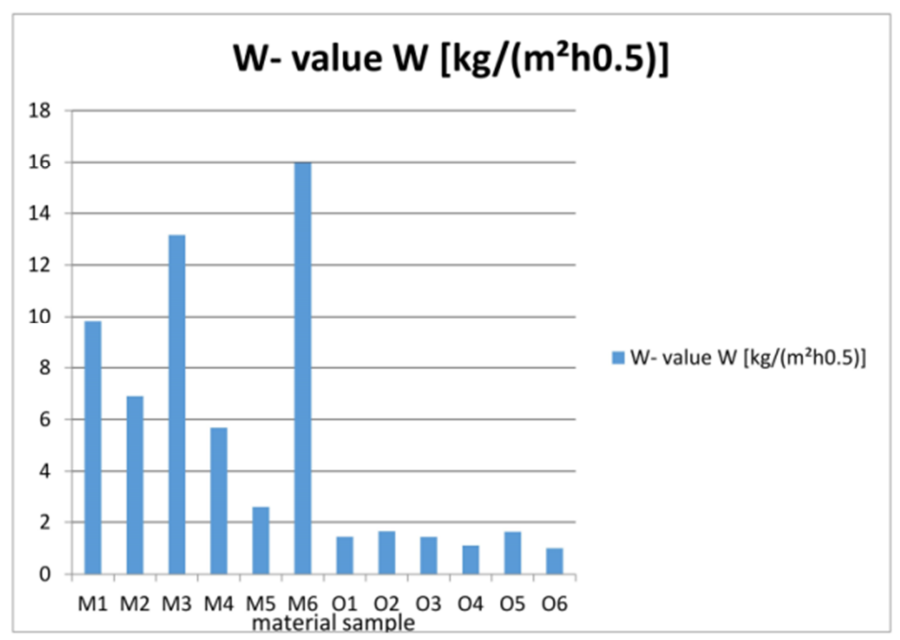

Fig. 4. Summary of water absorption coefficient

Pollack Periodica 14, 2019, 1 
The low rise diagram of water absorption can be seen in Fig. 5.

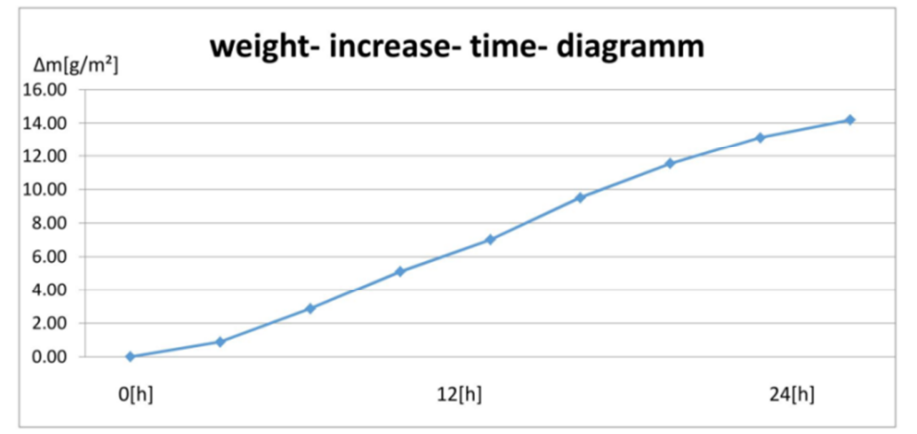

Fig. 5. Water absorption behavior of organically-bound plaster sample O5, $\Delta \mathrm{m}$ - weight increase of the area $\left[\mathrm{g} / \mathrm{m}^{2}\right] ; W$ value - $1.635\left[\mathrm{~kg} /\left(\mathrm{m}^{2} \mathrm{~h} 0.5\right)\right] ;$ (see Fig. 4)

High rise diagram of water absorption can be seen in Fig. 6 .

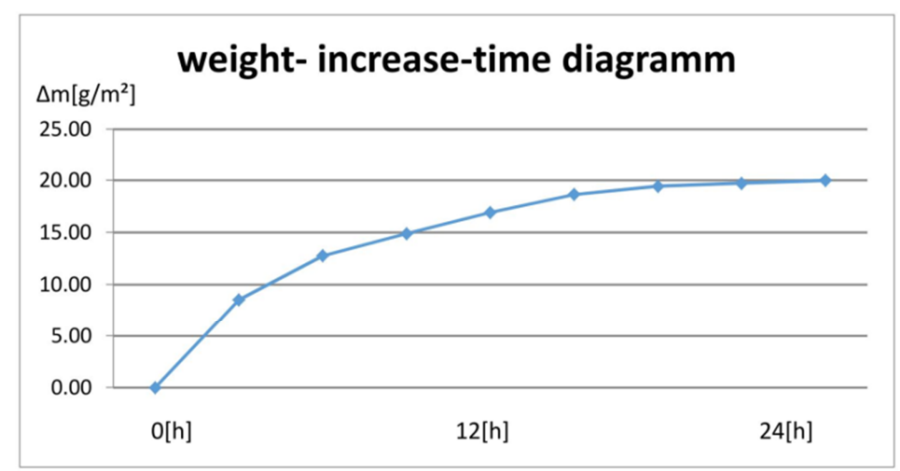

Fig. 6. Water absorption behavior of mineral bound plaster sample M2,

$\Delta \mathrm{m}$ - weight increase of the area $\left[\mathrm{g} / \mathrm{m}^{2}\right] ; \mathrm{W}$ value - $6.904\left[\mathrm{~kg} /\left(\mathrm{m}^{2} \mathrm{~h} 0.5\right)\right] ;$ (see Fig. 4)

Essentially, the following statement can be made: usually there is a higher water absorption capacity of mineral plasters in relation to organically bound plasters. This behavior ensures a better absorption of the moisture film in the upper layer of mineral plaster and prevents the formation of a film on the surface of the component. Fig. 7 shows the measured water quantity during $24 \mathrm{~h}$ absorption.

\section{Condensation water measurement:}

For an example of a condensation measurement in the free field test in Dreis (Germany) with suction fleece and electronic scales is presented in Fig. 8. The measurement procedure is only a static collection.

Fundamentally, a different strain of defrost water can be assumed in different locations as well as on different days. To achieve references it is necessary to have a 
similar uniform test as represented by a condensation chamber with an equally tempered test and an ultrasound vaporizer. Initial tests are in preparation.

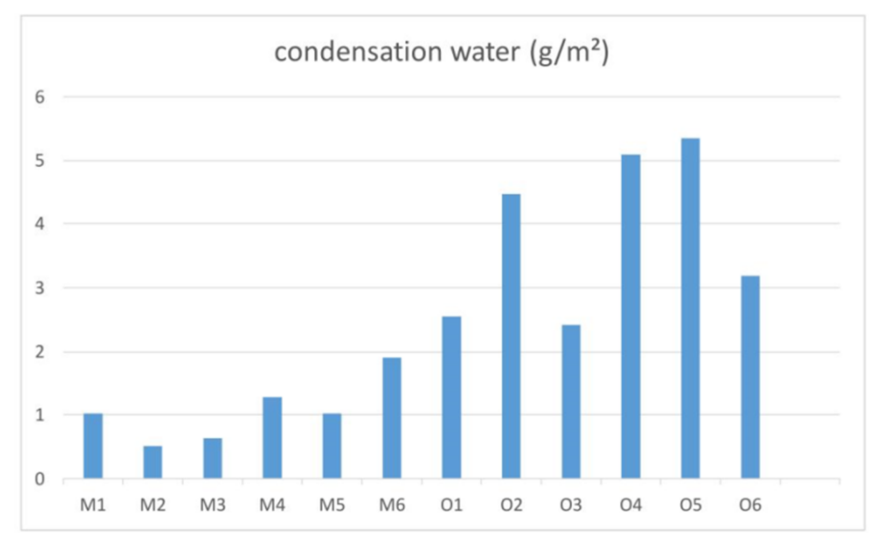

Fig. 7. Measured quantity of condensation water in one day in October 2017 (location: 54518 Dreis, Germany)

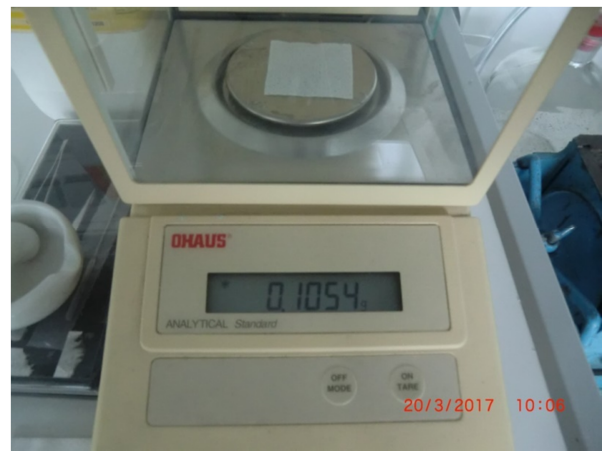

Fig. 8. Weighing of the suction fleece used to dab specimen of plaster surface

Another measurement technique is the LSM. Now there is a new method to carry out a dynamic analysis of condensation with a non-contact process.

Laser scanning technology allows a quantitative analysis of various characteristics, e.g. the surface composition and film thickness measurement of condensation. The light microscope is used for spatial representation. The LSM uses two paths with two light sources and two detectors. The white light source and the Charge-Coupled Device (CCD) camera chip produce a high-resolution microscope optical image. The second path consists of a laser diode. This provides the light source for capturing the height data due to the intensity of the reflective light. This is necessary for the height and length of peaks and valleys on a surface. The peaks and valleys are marked with different color thresholds on a scale; see Fig. 9 and Fig. 10. The LSM technology represents the metrological point of view in a dynamic process to measure the dew cycle 
of several materials and therefore first and foremost represents the possibility pictorially. Further questions that are fundamental refer to the 'behavior' of different materials of the facade surfaces. In the process, however it is very difficult to determine a comparative analysis between the samples due to their complex interaction air humidity and the influence of the temperature of the environment, as well as the weight of the sample itself.

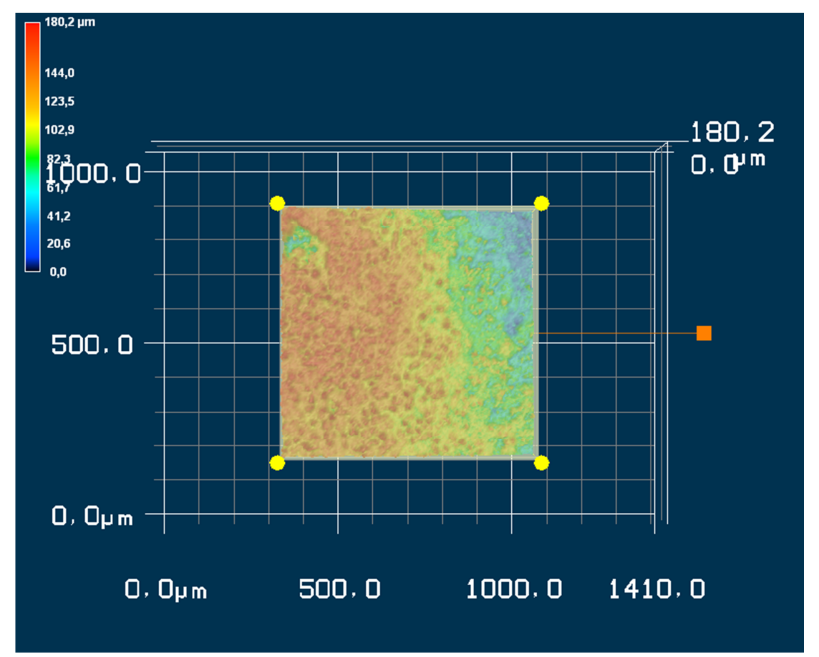

Fig. 9. Sample with organic plaster, example condensation time $=0$ minutes

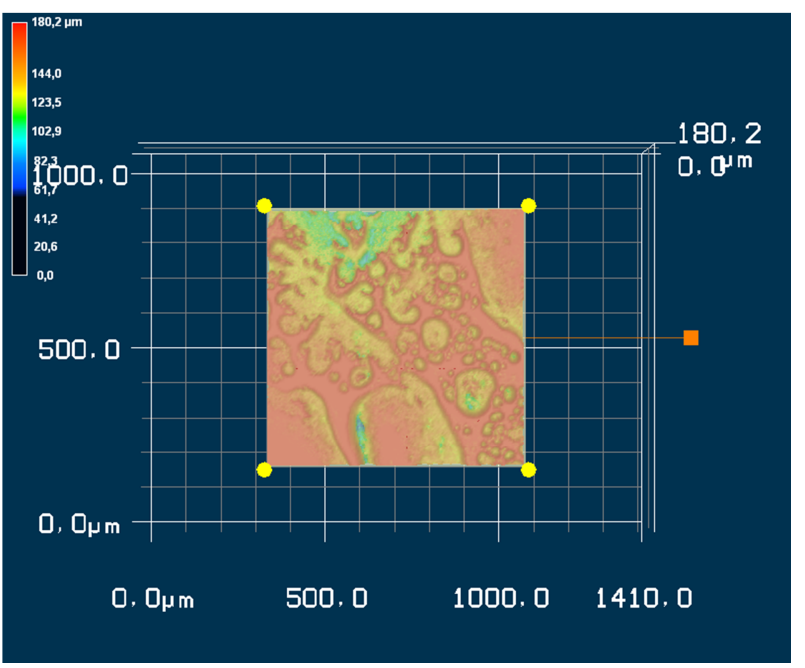

Fig. 10. Sample with organic plaster, example condensation time $=60$ minutes 
A comparison of deep contours with each other shows that the deep contours are almost completely filled. This effect of condensation water is metered by this new dynamic method.

Publication [12] by the author of this article has already appeared containing further descriptions of the LSM procedure for the measurement of condensation.

Condensation profile graphic in Fig 9 and Fig. 10 with the help of laser scanning microscopy describes the method in more detail. This analysis is only a comparison of samples; a comparative analysis is not possible due to the environmental chamber room [12, pp. 55-62].

\section{Conclusion}

The development of an exemplary representation for the future planning of moisture behavior of facades is a physical challenge. A model used to derive new and known parameters for condensation protection on a facade should improve condensation protection in the future. Due to the measurement procedures described in this article, it is now possible to determine a combination of good blow rain protection as well as good condensation protection. For example: an upper outer layer of plaster can allow accumulated condensate to evaporate again relatively quickly and a second layer of plaster is used for blow rain protection.

Further investigations into the constituent parts, e.g. additions and substitute products made from plastics (that will displace mineral ingredients due to lower purchasing costs) will generate new findings.

Materials science studies can offer further information to expand the capacity of different cleaning properties. A sustainable ecological design can make additional statements regarding surface protection without biocides.

Physical protection measures are to be preferred, because there is no impact on the environment from biocides.

\section{Open Access statement}

This is an open-access article distributed under the terms of the Creative Commons Attribution 4.0 International License (https://creativecommons.org/licenses/by/4.0/), which permits unrestricted use, distribution, and reproduction in any medium, provided the original author and source are credited, a link to the CC License is provided, and changes - if any - are indicated. (SID_1)

\section{References}

[1] Szodrai F., Lakatos A. Effect of the air motion on the heat transport behavior of wall structures, Pollack Periodica, Vol. 8, No. 1, 2017, pp. 67-73.

[2] Porhincak M., Estokava A., Vilcekova S. Comparison of environmental impact of building materials of three residential buildings, Pollack Periodica, Vol. 6, No. 3, 2011, pp. 53-62. 
[3] Donelly K., Mahle M., Chamberlain heights redevelopment: A large scale, cold climate study of affordable housing retrofits, Build America, US Department of Energy, 2012, https://www1.eere.energy.gov/buildings/publications/pdfs/building_america/chamberlain $\mathrm{h}$ ghts_redev.pdf, (last visited 10 March 2018).

[4] Buildings and climate change: status, challenges and opportunities, United Nation Environment Programme, https://www.unenvironment.org/resources/report/buildings-andclimate-change-status-challenges-and-opportunities, (last visited 10 February 2018).

[5] Kochin V., Wiehagen J. Construction guide, Next generation high performance walls, Build America, U.S. Department of Energy, 2017, https://www1.eere.energy.gov/buildings/ publications/pdfs/building_america/construction-guide-to-next-generation-highperformance-walls-in-climate-zones-3-5-part-2-2x4-walls_5.pdf, (last visited 4 March 2018).

[6] Wright G. S., Klingenberg K. Climate- specific passive building standards, Build America, U.S. Department of Energy, 2015, https://www1.eere.energy.gov/buildings/publications/ pdfs/building_america/climate-specific-passive-building.pdf, (last visited 3 March 2018).

[7] Venzmer H., von Werder J., Kogan D. Surface technology and preservation of structures, (in German) Beuth Verlag, Berlin, 2010.

[8] Künzel H. M. H., Fitz C., Krus M. Façade restoration, (in German), Beuth Verlag, Berlin, 2010.

[9] Burkhardt M., Vonbank R., Wash out of encapsulated biocides of façade, (in German) Schweitzer Federal Environmental Agency (BAFU) Bern, 2011.

[10] Kahle M., Nöh I., Biocide in waterbodies: Input and information to pollution and the effect, (in German) German Federal Environmental Agency, Dessau-Roslau, 2009.

[11] EN ISO 15148:2002, Hygrothermal performance of building materials and products Determination of water absorption coefficient by partial immersion, (in German) Beuth Verlag, Berlin, 2002.

[12] Venzmer H., Steffgen T. Energy supplier of old building, (in German) Beuth Verlag, Berlin, 2017. 